\title{
Migration, le bonheur ou un espoir idéalisé ? dans «Ulysse from Bagdad » d’Éric Emmanuel Schmitt
}

\author{
${ }^{1}$ Zeinab REZVANTALAB, ${ }^{2}$ Zahra HAJI BABAIE \\ 1,2 Université de Téhéran - Iran \\ Reçu le 20 mai 2018 | Accepté le 29 décembre 2018
}

\begin{abstract}
RÉSUMÉ. Cet article rapporte les résultats d'analyse d'« Ulysse from Baghdad », le roman d'Éric Emmanuel Schmitt, au point de vue de la question de migration. Nous nous appuyons sur les idées d'Abdelmalek Sayad (1933-1998), sociologue de l'immigration, pour répondre aux questions suivantes : Quel est le motif de la migration ? D'où vient le phénomène de la migration clandestine ? Quel est son impact sur la vie des réfugiés ? Comment cet effet socio-psychologique est-il présenté à travers une œuvre littéraire ? Quel rapport entretient Schmitt avec ce phénomène clé de notre temps ? Quelle est notre responsabilité face à ce phénomène ? On déduit qu'il faut avant toute chose donner à tout homme la possibilité de vivre dans le respect de sa dignité dans son pays. On constatera aussi que l'auteur a incité les candidats au voyage clandestin à s'éloigner des illusions et à décider avec le bon sens.
\end{abstract}

Mots-clés : Abdelmalek Sayad, Bonheur, Migration, Responsabilité, Schmitt.

\begin{abstract}
This article reports the analytical findings of Eric Emmanuel Schmitt's novel, "Ulysses from Baghdad", from the point of view of the issue of migration. We rely on the ideas of Abdelmalek Sayad (1933-1998), sociologist of immigration, in order to answer the following questions: What is the reason for the migration? Where does the phenomenon of clandestine migration come from? What impact can migration have on the lives of refugees? How is this socio-psychological effect presented through a literary work? What relationship does Schmitt have with this key phenomenon of our time? What is our responsibility for this phenomenon? It is deduced that it is necessary above all to give to every man the possibility of living in the respect of his dignity in his country. It will also be noted that the author has encouraged candidates to travel underground to get away from illusions and to decide with common sense.
\end{abstract}

Keywords: Abdelmalek Sayad, Happiness, Migration, Responsibility, Schmit.

田 auteur correspondant : z.rezvantalab@ut.ac.ir

Pour citer cet article (Style APA): Rezvantalab, Z. \& Haji Babaie, Z. (2018). Migration, le bonheur ou un espoir idéalisé? Dans "Ulysse from Bagdad" d'Éric Emmanuel Schmitt. Francisola : Revue Indonésienne de la langue et la littérature françaises, 3(2), 185-195. doi: 10.17509/francisola.v3i2.15752 


\section{INTRODUCTION}

La migration, phénomène aussi ancien que l'homme, est aujourd'hui d'actualité plus que jamais, en raison des conditions sociopolitiques ainsi que la multiplication des guerres en des régions comme Afrique noire, Asie centrale et Moyen-Orient. En outre, "dans le sillage des événements du 11septembre 2001, certains pays ont encore resserré leurs politiques à l'égard des immigrants, des réfugiés et des demandeurs d'asile. » (Dapsens ,2007) Ces mesures restrictives et la tendance majeure de nombreux gens pour la migration ont généré un grand problème : l'immigration clandestine. De ce fait, la migration s'est imposée aujourd'hui comme l'un des objets centraux de la sociologie au point qu'elle constitue un champ de recherche à part entière. Des sociologues comme Abdelmalek Sayad ont tenté de dévoiler des faces cachées de la migration. Sayad (1999) a analysé avec un grand soin la situation embarrassante dans laquelle se trouve l'émigré-immigré. Les nombreux articles consacrés à ces analyses ont été rassemblés dans son livre « La double absence, Des illusions de l'émigré aux souffrances de l'immigré ».Les dits et les non-dits de la condition d'immigré sont présents dans ce livre. Les modèles théoriques qu'il a élaborés possèdent une pertinence à même d'aider à comprendre les émigrations les plus diverses et à penser les situations des immigrés et des sans papier.

Par ailleurs, comme la littérature et la société sont deux éléments inséparables qui influent l'une l'autre, le phénomène de la migration a ainsi trouvé une expression privilégiée dans la littérature. Des études intéressantes ont été effectuées à propos de répercussions de ce sujet dans la littérature, notamment celle de Christiane Albert, pour qui l'immigration est « un discours qui produit ses propres modalités d'écriture » (Albert, 2005, 19). Ou encore, la recherche d'Anne Schneider, concernant la littérature jeunesse migrante, qui voit là une «visée testimoniale » (Schneider, 2013, p. 24).

On témoigne de la peinture de ce phénomène universel chez des romanciers comme Tahar Ben Jelloun, dans la deuxième moitié des années 70, Azouz Begag («Le gone du chaaba », 1986) et Leila Houari (« Zeida de nulle part »,1985) dans les années 80. Cependant, la plupart de ces romanciers relèguent au second plan la question de l'immigration clandestine. De ce fait, dans le cadre de ce travail, nous avons essayé de choisir un romancier qui s'attache à la peinture de l'émergence des filières d'immigration clandestine : il s'agit d'Éric Emmanuel Schmitt. Ce dernier dans son « Ulysse from Bagdad ", à travers le statut d'un jeune irakien, appelé Saad, qui fuit son pays pour quitter le chaos, dénonce les violences psychologiques et les humiliations faites aux immigrés en situation irrégulières.

Malgré la pluralité des recherches sur la thématique de la migration dans la littérature française, on note que ce roman de Schmitt qui doit surtout sa particularité par le fait qu'il délègue la parole à un immigré qui nous étend la trame de l'histoire de sa vie, est encore peu analysé. Ce roman, loin d'être une évocation figée et restreinte dans le genre de la littérature réaliste, rend la problématique de l'immigration assez concrète et nous permet d'analyser ce phénomène. Comme l'affirme Gisèle Sapiro, « la signification que prennent les œuvres est inséparable de leur interprétation et des appropriations dont elles sont l'objet " (Sapiro, 2007, 2).En nous appuyant sur une analyse descriptive, nous essayons de répondre à ces questions : Quel est le motif de la migration ? D'où vient le phénomène de la migration clandestine ? Quel impact peut avoir la migration sur la vie des réfugiés? Comment cet effet sociopsychologique est-il présenté à travers une œuvre littéraire ? Quel rapport entretient Schmitt avec ce phénomène clé de notre temps qu'est la migration ? Quelle est notre responsabilité face à ce phénomène?

Afin de donner une réponse à ces questions de recherche, nous nous appuyons sur les idées de Sayad (1999) qui relève de trois principes. Le premier dit $\mathrm{qu}^{\prime}$ « avant l'immigration il y a l'émigration : «émigration et immigration deux phénomènes aussi indissociables que le recto et le verso d'une 
même feuille ». Le second principe est que « les États sont parties prenantes » dans les émigrations -immigrations avec la domination des États les plus forts sur les États dépendants. Le troisième principe permet de comprendre que ce phénomène est organisé autour de la dissimulation de la réalité à ses propres yeux comme à ceux des autres ici et là-bas, et autour des «illusions » du retour.

Selon le thème de notre roman, nous basons notre recherche sur le premier principe exprimé par Sayad dans son livre. Sayad a porté un double regard sur la question de l'immigration, il a placé au cœur de son analyse l'émigré-immigré:

Immigration ici et émigration là sont les deux faces indissociables d'une même réalité, elles ne peuvent s'expliquer l'une sans l'autre: c'est dans un même mouvement, dans une même perspective " historico-culturelle ", selon la même logique mais aux deux extrêmes du même processus, que s'engendrent, se perpétuent et se résolvent les deux dimensions, émigration et immigration, du même phénomène. » (Sayad, 1984, p. 302)

Adopter ce principe a conduit Sayad à mener des études historiques sur la colonisation de l'Algérie, puis sur les conditions des immigrés. De même, nous traiterons d'abord les caractères de l'ambiance socio-culturelle dans laquelle vivent les émigrants et les immigrants pour trouver la réponse de deux premières questions de notre recherche.

Par ailleurs, Sayad ne considère plus l'immigration en termes de coûts et avantages économiques, il a jeté de cette façon, un regard humain sur la question délicate des migrations et ainsi, sa sociologie vient en rupture avec les analyses sociologiques précédentes qui portaient un regard très déshumanisant sur l'immigré. Il montre que l'émigration-immigration est une véritable déchirure spirituelle, une souffrance individuelle. S'inspirant de ces approches théoriques, nous montrerons le processus de passage des illusions de l'émigré aux souffrances de l'immigré à travers les personnages de notre roman. Et de cette façon, nous pouvons répondre aux questions de recherche.

\section{MÉTHODE}

Dans la présente étude, nous nous appuyons sur une analyse descriptive qui nous permet de traiter minutieusement le phénomène de la migration. La donnée de cette recherche est le roman de Schmitt, " Ulysse from Bagdad ". Comme cette recherche se concentre sur les idéologies exprimées dans notre contexte, sur le phénomène de la migration, nous commençons d'abord par l'analyse des discours des personnages immigrés de notre roman pour comprendre la manière dont l'écrivain a choisi pour manœuvrer sur ce phénomène ensuite nous essayons de trouver ,à travers les discours des personnages, les idéologies de l'auteur en tant qu'un philosophe sur ce phénomène mondial, puis pour collecter des données sur la problématique de migration tout en nous référant à la bibliothèque de faculté de langues et littérature étrangère de l'université de Téhéran et à la médiathèque de l'ambassade de France en Iran, nous consultons aux sites suivantes en recherche d'informations efficaces sur ce sujet :Open Edition et Erudit qui sont des portails de ressources électroniques en science humaine et sociales et aussi Theses.fr qui propose l'accès aux thèses françaises. Voici les mots à travers lesquels nous avons tenté de trouver des informations sur notre sujet dans la littérature : la migration dans la littérature, la migration clandestine, l'identité des immigrés, la question de migration chez Éric Emmanuel Schmitt.

Pour arriver aux résultats escomptés, nous divisons notre étude en trois parties : tout en nous appuyant sur les idées de Sayad (1999) tout au long de notre recherche, nous analysons d'abord, le pourquoi du départ du personnage principal vers lointain, ensuite nous montrons les interactions de notre héros devant les obstacles qui se dressent devant lui et à la fin, nous relevons comment notre héros, plein d'espoir de trouver le bonheur l'ailleurs est atteint de crise d'identité. 


\section{RÉSULTATS ET DISCUSSION}

\subsection{Un Voyage vers l'avenir}

En tant que romancier et philosophe, Schmitt combine habilement la réalité et la fiction, la question sociale et l'idéologie philosophique, le passé et le présent, l'orient et l'occident. En fait, ce roman donne au lecteur un aperçu sur les grands événements qui ont marqué l'Histoire de l'Irak, sur la façon dont la guerre et l'invasion de l'Irak par les forces américaines ont détruit la culture autochtone et le style de vie des irakiens. "Ulysse from Bagdad », constitué de 15 parties, commence par un flash-back au passé du personnage-narrateur. Celui-ci décrit, dans un premier temps, la dictature et l'injustice que subissait l'Irak en raison des problèmes raciaux et religieux (Saddam proclamait sa haine à l'encontre des Kurdes, Juifs, Chiites) sous la tyrannie de Saddam Hussein. Il peint la dégradation des conditions de vie des irakiens par les faits et la politique agressive du Président. Il nous raconte comment il a vu mourir, un à un, la plupart des siens, surtout son père. Après le meurtre de celui-ci par les américains, Saad, devenu responsable de sa famille, doit abandonner ses études pour subvenir aux besoins de sa famille.

On peut considérer l'histoire de la vie de Saad et sa famille comme le microcosme de celle de la vie de tous les irakiens. Ce qui est arrivé à Saad est aussi arrivé sous différentes formes à la plupart des jeunes irakiens. Cela montre que l'Irak est devenu le lieu chaotique de toutes les souffrances. En effet, la trame des événements narrés par Saad nous montre comment suite à la déclaration de la guerre par Saddam Hussein contre le Koweït en août1990, l'Irak a été placé sous embargo, ce qui a causé la famine et la ruine. Nous constaterons que le désastre des irakiens ne s'arrête pas là. Après la chute de Saddam, malgré la pensée des irakiens, rien n'a changé, ils sont même plongés dans une situation de plus en plus mauvaise : le terrorisme et les exactions de toutes sortes sous l'invasion de leur pays par les forces américaines. Tout cela montre au lecteur comment l'Irak est devenu comme un enfer aux 20e et $21 \mathrm{e}$ siècles. Ces conditions ne permettent pas à Saad de bien travailler, ni de gagner de l'argent pour aider sa famille. C'est ainsi qu'il ne peut plus rester dans son pays en ruine, dévasté par la guerre et appauvri par l'embargo. Ainsi écoutant sa mère, il veut fuir Bagdad. Dans cette perspective, la décision de partir demeure rationnelle; elle ne découle pas d'une attitude marginale ou exclusivement rêveuse. Elle s'avère cependant la « seule solution raisonnable » (Gauthier, 1997, p. 315). Désormais, il apparaît comme un voyageur qui doit se confronter à des contraintes, un Ulysse quittant Bagdad pour rejoindre Londres, ce qui est annoncé dès le début par le titre : "Ulysse from Bagdad ». De cette façon, Saad entreprend un long voyage périlleux. Il s'éloigne de ses proches pour s'évader d'un pays à feu et à sang, et pour s'inventer un avenir en Grande-Bretagne. Son acte s'associe ainsi à un héroïsme qu'il assume en se désignant comme un aventurier.

Le recours de l'auteur à l'arrière-plan socio-culturel, le prêt de la parole à un démuni et le traitement des informations historiques ont permis à l'auteur d'exprimer au premier étape sa pensée philosophique sur des questions sociaux d'une manière implicite. Par exemple en suivant les dialogues entre Saad et son père, on peut souligner la pensée philosophique de Schmitt sur la guerre : « - Alors d'où viennent les guerres ? - L'origine des conflits, c'est le « nous », ce « nous » d'une communauté contre une autre, ce «nous » exprimant une identité et justifiant d'attaquer les identités étrangères (Schmitt, 2008, p.120).

Et en deuxième étape, cela lui permet de critiquer la politique dominante en Irak en tant que le pays de départ pour mieux justifier les causes du départ de jeunes comme notre héros. En effet, l'auteur à travers la présentation des causes du départ de Saad a réussi à souligner que les phénomènes migratoires dépendent en grande partie du non-respect des droits de l'homme et du manque de la démocratie dans les pays d'où viennent les populations 
migrantes. Tout individu a droit à la vie, à la liberté et à la sureté de sa personne. Mais, en Irak, il n'a jamais existé de démocratie qui puisse garantir les droits fondamentaux de la personne humaine et il n'y avait pas une loi qui s'opposait à toute forme de tyrannie ou de dictature ne respectant pas les droits de l'homme. C'est cela qui pousse les gens de ces types de pays à la quête du bonheur ailleurs. Mais la quête du bonheur, la recherche de l'Eldorado n'est pas aisée. Devant l'espoir de partir vers un avenir meilleur, se dressent toujours beaucoup d'obstacles. Dans notre deuxième partie, nous allons voir à travers le personnage de Saad l'interaction des émigrants devant les obstacles.

\subsection{Interaction sujet-norme}

Notre Ulysse tout comme le roi d'Ithaque sera confronté aux maintes aventures : la difficulté de partir, les dangers de la mer, la survie difficile et les horribles conditions de voyage. Le premier obstacle devant lui c'est le manque d'argent pour son voyage : « je n'ai pas réuni la somme, ni pour le voyage ni pour m'installer à Londres. " (Schmitt, 2008, p. 38). Sayad dans son livre a exprimé ce problème à travers les discours des immigrés qui ont fait tout pour arriver à l'argent. En effet, une telle situation est susceptible de pousser l'émigrant à la délinquance. Comme on l'a montré, Saad n'a pas la possibilité de vivre dignement dans son pays et de l'autre côté, il n'a même pas un dinar pour le quitter. De cette façon, la volonté de partir le pousse à adapter un comportement différent de celui dont il s'attend de lui-même; il entre dans une bande de trafic et transporte " une cargaison de drogue » au Caire. La représentation de cette situation dans le roman s'annonce comme un alibi pour critiquer le système dominant dans ce pays qui est indifférent aux droits de son peuple et les pousse ainsi vers l'abime. Les obstacles qui s'étendent devant l'immigrant sont à un nombre infini. Selon Sayad, le deuxième obstacle ou peutêtre le plus difficile devant le candidat à l'immigration est l'obtention de l'autorisation de sortie. C'est ce que le lecteur constate chez Saad ; au Caire, Saad doit s'attendre six mois pour avoir un rendez-vous au « HautCommissariat des Nations unies pour les réfugiés ». Ce qui rend difficile cette attente pour lui c'est que pendant ce temps-là, il n'a le droit ni de louer un lieu pour vivre, ni de travailler. De cette manière, il doit consentir à un modeste travail ; soit de faire le gigolo dans un dancing. Schmitt pour mettre en question les lois établies pour donner le statut de réfugié à un immigrant prête la parole à Boubacar, l'ami africain de Saad au Caire. Au jour de rendez-vous Boubacar donne ces conseils à Saad pour le préparer à l'entretien :

[...], si on leur explique qu'on fuit la pauvreté, qu'on veut décrocher un travail et envoyer de l'argent à sa famille pour qu'elle survive, on ne les intéresse pas. Ils ont besoin de spectacle, de scandales politiques, de massacres, de génocides, de dictateurs levant des armées de salauds maniant la machette ou la mitraillette » (Schmitt, 2008, p.62 - 63)

Schmitt montre ainsi comment ces autorités officielles demandent à entendre seulement des mensonges pour leur profit personnel. Les déclarations de Boubacar se justifient au moment où on refuse à Saad le statut de réfugié. En effet, Saad s'est contenté de dire la vérité et il a évité toute sorte de tromperie : «- Je ne tricherai pas d'un seul mot. Si j'ai quitté l'Irak, c'est parce que je suis à la recherche d'une vie droite, sans compromis. » (Schmitt, 2008 :63) Le statut de réfugié lui est refusé sous prétexte que l'Irak a été libéré par les Etats-Unis d'Amérique : « - [...] Parce que l'Irak est un pays libre aujourd'hui. Parce que l'Irak roule vers la démocratie. Il n'y a donc plus de problème. " (Schmitt, 2008, p.66) Le narrateur montrent le décalage qui existe entre le vécu des irakiens et la vision un peu simpliste de ces gens : " Selon eux: ils ne nous ont pas envahis, ils nous ont délivrés ; ils n'ont pas créé la confusion dans notre pays, ils butent sur des Irakiens incapables de recevoir la paix. " (Schmitt, 2008, p.68) Cela reconfigure une nouvelle dialectique entre le même et l'autre. En effet, on demande le retour de ces immigrants à leur pays, tandis que le retour pour eux est une catastrophe :

[...], ils n'imaginaient pas que nous transportions avec nous les espoirs de 
nos proches, non, ils avaient le sentiment d'avoir accompli leur devoir, pas d'avoir bousillé trente vies, et derrière ces trente vies, trente familles, soit deux cents ou trois cents personnes, qui comptaient sur nous. (Schmitt, 2008, p.110)

Schmitt, tout comme Montesquieu dans les Lettres persanes, en prêtant la parole à des étrangers, critique les occidentaux. Il met en question la politique frontalière de l'Europe qui non seulement contrevient aux libertés fondamentales de l'individu mais qui, à travers une série de mesures tend à restreindre, puis à criminaliser les déplacements de la génération de tout un continent. Maintenant que le statut de réfugié lui est refusé, Saad apparaît aux yeux des autres gens comme un immigré irrégulier. Cette situation irrégulière le fige aussi dans une dénomination, le « clandestin » ou le «sans-papiers » qui est stigmatisé et criminalisé. C. Mazauric précise à ce sujet que : " du point de vue du droit international, ce qui est criminel, ce n'est pas le fait, pour un individu, d'émigrer c'est le fait, pour une autorité publique, de l'en empêcher. »(2012:11) De la même manière, Saad, lui-même ne se considère pas comme un criminel : "Je suis un cas non prévu par la loi, mais pas contre la loi. » (Schmitt, 2008, p. 112), mais simplement comme un voyageur qui, contraint par une politique d'immigration trop rigide, essaie d'échapper au contrôle des garde-frontières.

Nous le voyons faire face à la difficulté de franchir les frontières et aux politiques d'immigration des pays comme l'Egypte, la Lybie, la Tunisie, l'Italie et la France, dans lesquels il débarque. En guise d'exemple, on peut citer son arrestation dans un centre de rétention à Malte, qu'il doit fuir comme Ulysse par la mer en affrontant les tempêtes. C'est au cours de ce voyage qu'il perd son ami, Boub. On peut encore faire allusion au moment où après la réalisation d'un dur travail pendant plusieurs mois, il veut franchir la frontière d'Italie mais les douaniers italiens l'arrêtent au moment de partir :

Les douaniers - et les journalistes à l'unisson - se réjouissaient d'avoir intercepté notre camion; ils se flattaient de nous avoir arrachés à un voyage dégradant, [...], comme des chiens errants, on nous destinait à des refuges une fourrière - ; certains d'entre nous seraient rendus à leur maître - leur pays - si on les identifiait. (Schmitt, 2008, p.110)

Ce comportement hypocrite est déjà annoncé par les discours de Saad et le fantôme de son père qui sont chargés de lourdes critiques aux européens :

Tu sais, Saad, la dictature, au moins c'est clair, ça joue franc jeu : on sait qu'il y a un pouvoir central, total, qui exerce son arbitraire en toute impunité. En Occident, $c^{\prime}$ est plus vicieux : pas de despote mais des administrations bloquées, des règlements plus longs que tous les annuaires téléphoniques, des lois concoctées par des êtres bien intentionnés. (Schmitt, 2008, p.69)

Mais Schmitt ne se contente pas seulement de l'analyse des difficultés de franchir les frontières de l'Europe, il s'attache aussi à l'appréhension du vécu de l'immigré en situation irrégulière dans l'espace européen. A ce propos, Sayad souligne dans son livre que l'immigré a le sentiment de l'isolement et de l'exclusion du pays d'arrivé qui le traite comme simple force de travail. A .Manço Altay aussi dans son livre, « Processus identitaires et intégration ", souligne l'idée de Touraine que « les migrants souvent souffrent d'une manière profonde des phénomènes d'exclusion, d'insécurité et de ségrégation. " (Manço, 2006, p.56). De même, Schmitt montre la forme d'exploitation dont les immigrés en situation irrégulière sont l'objet de la part de certains des employeurs, à la recherche d'une main d'œuvre bon marché. En outre, en attribuant le statut d'un étudiant à son personnage immigrant, il dénonce que l'immigrant venu n'est pas forcément l'illettré ou le laissé-pour compte que le préjugé et les médias contribuent à façonner en Europe. Il pourrait devenir au contraire une personne qui a la capacité de s'épanouir si on lui donne l'occasion.

Sayad, à travers ses recherches et ses analyses, montre que les immigrés, victimes d'effets continus de ségrégation et de discrimination, sont voués à travailler dans les secteurs les plus dévalorisé de l'économie du pays d'arrivée. Ce que l'on constate dans 
le contexte de notre étude c'est la concrétisation des théories de Sayad. " Ulysse from Bagdad » apparaît comme un témoignage sur la condition de l'immigré. Cela nous permet d'avoir une appréhension du vécu de l'immigré en situation irrégulière.

Tout au long du roman, le narrateur dans le statut d'un immigré manifeste le décalage entre son rêve et la réalité ; un jeune garçon qui était dès sa naissance promis à un bon avenir, a dû alors avoir un statut de clandestin et occuper une place inférieure: « je n'imaginais pas que, sitôt que je débarquerais dans le monde non arabe, je deviendrais 1'Arabe de service. " (Schmitt, 2008, p.71)

La mise en fiction de l'état de vie de ce démuni, pourrait être considérée comme une critique aux responsables de la provocation de ces mauvaises conditions chez la plupart des immigrés :

Je pense que, derrière la porte, mon destin m'attend. La femme qui va $\mathrm{m}^{\prime}$ interroger - $[. .$.$] - est une magicienne$ qui tient ma vie entre ses mains. Selon ce qu'elle pensera de moi, elle deviendra fée ou sorcière, bonne ou cruelle, car elle a le pouvoir de me métamorphoser en avocat anglais ou en porc vautré dans sa souille. (Schmitt, 2008, p.66)

Saad en tant qu'étudient du droit aurait pu acquérir une place privilégiée, mais depuis qu'on lui a refusé le statut de réfugié, il n'est qu'une sorte d'esclave : «Tu vas même devoir travailler beaucoup pour manger peu. » (Schmitt, 2008, p.58)

Chez Saad, il existe un décalage entre le travail intellectuel auquel il pensait et le travail manuel auquel il est destiné. Il est même soumis à des traitements qui le mettent au rang de bestiaux : «Certains jours, j'ai l'impression de devenir étranger à l'espèce humaine... » (Schmitt, 2008, p.58). Son travail a dégradé non seulement son statut social mais aussi sa santé physique : « [...], mais as-tu vu tes mains ? Entaillées. Déchirées. » (Schmitt, 2008, p.106) En effet, au lieu de continuer ses études à l'étranger, il a été sous l'emprise des mafieux dans l'espoir de pouvoir franchir les frontières :

Contre quatre à six mois d'un salaire courant, ils assuraient le transfert jusqu'à la mer du Nord, [...] », « Toujours moderne, à l'affût des nouveaux marchés ! La mafia a pressenti qu'il y a de l'argent à gratter auprès des clandestins. Tel est le génie $\mathrm{du}$ commerce, mon fils : comprendre qu'on peut tirer autant d'or des pauvres que des riches. " (Schmitt, 2008, p.105)

Ces réseaux mafieux sont, en effet, représentatifs de nouvelles formes d'esclavagisme, tirant profit de la situation irrégulière de ces immigrants. Pour eux, un immigrant est essentiellement conçu comme une force de travail, qui ne leur coûte pas cher. Ainsi Saad est-il devenu le symbole des individus dont l'existence est maintenue dans une impasse par un ordre géopolitique injuste:

A la loterie de la naissance, on tire de bons, de mauvais numéros. Quand on atterrit en Amérique, en Europe, au Japon, on se pose et c'est fini : on naît une fois pour toutes, nul besoin de recommencer. Tandis que lorsqu' on voit le jour en Afrique ou au Moyen-Orient... (Schmitt, 2008, p.6)

Ces déclarations sont la preuve des travers d'un système où les humains n'ont pas les mêmes droits et les mêmes chances :

[...] en face de moi, ils (les occidentaux) réalisent qu'ils ont de la chance, qu'ils ont tiré un bon numéro, que le couperet fatal leur est passé au ras des fesses, et se souvenir de cette première et constitutive fragilité les glace, les paralyse. (Schmitt, 2008, p.115)

Le privilège qu'a l'histoire de Saad c'est qu'il représente le phénomène de la migration sous différents angles : d'abord comme on l'a montré, du point de vue d'un immigré, ensuite du point de vue de ceux qui dans les pays d'accueil sont en contact avec l'immigré:

Moi, le clandestin, je leur rappelle cela. Le vide. Le hasard qui les fonde. A tous. C'est pour ça qu'ils me haïssent. Parce que je rôde dans leurs villes, parce que je squatte leurs bâtiments désaffectés, parce que j'accepte le travail qu'ils refusent, je leur dis, aux Européens, que j'aimerais être à leur place, que les privilèges que le sort aveugle leur a donnés, je voudrais les acquérir. (Schmitt, 2008, p.115)

En outre, certains dévisagent les immigrants clandestins comme des êtres inférieurs, des sous-hommes qui détiennent 
moins de droits que les autres, mais l'auteur, à travers le dialogue de Saad avec son père, a essayé d'alerter ses lecteurs et de les pousser vers la correction de leur comportement :

Papa, qui sont les barbares ? Ceux qu'on estime inférieurs ? Ou ceux qui s'estiment supérieurs ? -C'est là que commence la barbarie, Saad : quand on ne se reconnaît plus dans l'autre, quand on désigne des sous-hommes, quand on classe l'humain de façon hiérarchique et qu'on exclut certains de l'humanité. " (Schmitt, 2008, p.128)

Ce genre de dialogues nous donne l'impression d'assister à des discours philosophiques éclaircissant de meilleure façon et sans aucun préjugé les questions concernant ce phénomène actuel. Dans notre contexte, maints sont les exemples qui montrent ces dialogues philosophiques. En guise d'exemple le dialogue de Saad avec l'officier de la douane déclare les idéologies philosophiques de l'auteur sur la question du passage des frontières, l'obstacle auquel doit se confronter un immigrant :

Mais on a bien le droit de se demander pourquoi elles existent. Et sont-elles une bonne solution aux problèmes humains? Eriger des frontières, est-ce la seule manière pour les hommes de vivre ensemble?

- Jusqu'ici il n'y en a pas eu d'autre.

- Même si c'est la seule manière, est-ce la bonne ? L'histoire humaine, c'est l'histoire de frontières qui se déplacent. Qu'est-ce que le progrès sinon la raréfaction des frontières ? [...]

-Les gens ont quand même besoin de dire « nous » pour exister : nous les Américains, nous les Africains, nous les Européens.

- Ne peuvent-ils pas essayer «nous les hommes » ? S'interrogea l'officier.

- Alors ce serait contre les animaux.

- Dans ce cas-là, pour les inclure encore, ils pourraient tenter « nous les êtres vivants »? (Schmitt, 2008, p.112-113)

L'auteur essaie ainsi de propager des idées humanistes concernant la solidarité. Il montre à ses lecteurs qu'il faut évoluer le regard porté sur ces démunis parce que chaque individu aurait pu être à la place de ces clandestins. Et parfois en faisant tenir à des étrangers des propos sévères envers les responsables, il dénonce à ceux-ci d'une manière oblique les aspects négatifs de leur comportement. En guise d'exemple, on peut faire allusion à l'incohérence entre leur parole et leur action, ce qui met au jour leur hypocrisie:

Ils parlent de paix et ils font la guerre, ils créent de la rationalité et tuent à tour de bras, ils inventent les Droits de l'homme et ils totalisent le plus grand nombre de vols, d'annexions, de massacres de toute l'histoire humaine. Drôle de peuple, les Européens, l'ami, drôle de peuple, un peuple dont la tête ne communique pas avec les mains. » (Schmitt, 2008, p.112-113)

Schmitt ne provient pas socialement des sphères de l'immigration, mais ayant été sensibilisé à cette question. Par le prêt des propos critiques et philosophiques sur cette problématique à ses personnages, il a essayé de changer les regards portés sur les immigrés et d'aider ainsi à l'amélioration des conditions de vie de ces hommes. Selon le mot de Sartre : «L'écrivain est en situation dans son époque : chaque parole a des retentissements. Chaque silence aussi. » (Sartre, 1948, p.13)

La dénonciation de toutes les injustices pourrait être considérée comme un préambule pour montrer les effets néfastes de la situation irrégulière des immigrés sur leur personnalité et leur psyché. En effet, de tout ce qui constitue la vie des clandestins : la faim, la pauvreté, les interrogatoires, les passages à tabac, l'humiliation, le racisme, ce qui semble le plus insupportable c'est le déchirement identitaire : « Je(Saad) n'appartiens à aucune nation, ni au pays que j'ai fui ni au pays que je désire rejoindre, encore moins aux pays que je traverse. Clandestin. Juste clandestin. Bienvenu nulle part. Etranger partout. » (Schmitt, 2008, p.6) Les comportements de Saad relèvent les traces du malaise identitaire chez un clandestin et nous permettent de revendiquer les troubles de l'identité dans la partie suivante de notre recherche.

\subsection{La crise d'identité}

La question d'identité traverse toujours le phénomène de l'immigration. La plupart des chercheurs et des sociologues qui se sont penchés sur ce phénomène admettent que l'une des questions fondamentales est celle de l'identité. Sayad a formulé ce problème en tant que le problème de 
l'intégration, il la considère comme un processus qui consiste, idéalement, à passer de l'altérité la plus radicale à l'identité la plus totale. (Sayad, 1999, p. 307). Par exemple, Saad qui représente un être ayant rompu avec son passé dans l'espoir de trouver le bonheur ailleurs, perd peu à peu non seulement son identité sociale mais aussi son identité personnelle. Il trouve une identité plurielle et même complexe : « réclamant le statut de réfugié, j'ai dégringolé d'identité en identité, migrant, mendiant, illégal, sanspapiers, sans-droits, sans-travail ; le seul vocable qui me définit désormais est clandestin. » (Schmitt, 2008, p. 6)

Dans notre roman, la question d'identité est envisagée dans une perspective critique. Saad est représentatif d'un immigré qui n'avait aucun problème avec son identité sociale et personnelle avant le départ. Il était très satisfait de son identité arabe, et il n'avait aucun problème avec sa famille. Son seul problème était le lieu auquel il appartenait, un lieu qui ne lui permettait pas de vivre à l'aise, il voulait changer son destin sans perdre son identité:

Souvent je rêve d'avoir été avant d'être, [...] : alors je corrige, je guide la roue qui brassait les cellules, les molécules, les gènes, je la dévie afin d'en modifier le résultat. Pas pour me rendre différent. Non. Juste éclore ailleurs. Autre ville, pays distinct. Même ventre certes, les entrailles de cette mère que j'adore, mais ventre qui me dépose sur un sol où je peux croître, et pas au fond d'un trou dont je dois, vingt ans plus tard, m'extirper. » (Schmitt, 2008, p.6)

Mais on constate comment la difficulté de franchir les frontières l'a obligé à être coupé de ses éléments identitaires. Pour éviter d'être retourné à son pays, Saad doit taire son identité : «Depuis quelques années, j'ai subi je ne sais combien d'entretiens comme celui que vous allez $\mathrm{m}$ 'infliger et cela n'a jamais servi à rien. Je dois mal répondre puisqu'on me ferme toujours la porte. " (Schmitt, 2008, p.111). C'est ainsi qu'il se présente lors de ses entretiens : « - Ulysse. Parfois aussi je m'appelle Personne. " (Schmitt, 2008, p .111) Depuis son glissement dans le monde des clandestins, il ne peut plus faire confiance à personne : « un univers dont le ciment est la peur ; chacun se révèle suspect, celui qui porte un uniforme, celui qui n'en porte pas [...]» (Schmitt, 2008, p.85). Les mauvaises conditions de sa vie ont aussi influencé son identité morale, Saad qui montrait toujours de l'honnêteté et évitait même un petit mensonge, doit maintenant fermer les yeux sur la morale : « Dès le premier larcin, je mis ma morale entre parenthèses. [...], je ne songeais jamais aux victimes, aux entreprises spoliées, aux industriels dépouillés, encore moins aux particuliers découvrant leurs maisons sans toit. Je travaillais dur, gagnais peu, serrais les dents. » (Schmitt, 2008, p.106). Tout cela crée un écart entre l'identité acquise et l'identité visée. Les conditions misérables de sa vie l'ont assigné à la marge, il est spatialement destiné à des lieux non habitables : « une ruelle puante », « une bouche d'égout », « en marge d'un terrain vague, non loin d'une décharge à ordures, une ruine d'au moins un siècle où, occupant le troisième étage, lui et d'autres Libériens avaient installé leurs sacs, des matelas de récupération, un vieux réchaud à gaz. C'était sale, malodorant, exigu et chaleureux. " (Schmitt, 2008, p.58) ou confiné dans des sites qui s'apparentent à ce que Foucault nomme des " hétérotopies » : « Au-delà de la ville et des villages, perdu dans la campagne boueuse, le squat consistait en d'anciens bâtiments administratifs et d'exlogements ouvriers désaffectés depuis la faillite du site. Les clandestins l'avaient colonisé en espérant que son éloignement favoriserait une relative tranquillité. » (Schmitt, 2008, p.127)

Notre Ulysse n'est reconnu nulle part :

Comme Socrate selon Platon, l'immigré est atopos, sans lieu, déplacé, inclassable [...] Ni citoyen, ni étranger, ni vraiment du côté du Même, ni totalement du côté de l'Autre, il se situe en ce lieu «bâtard » dont parle aussi Platon, la frontière de l'être et du non-être social. (Sayad, 1999, p. 12)

Non seulement, Saad n'a aucune place, mais encore il n'a aucun droit, même le droit de savoir la raison de ses souffrances. Cette difficulté de s'affirmer en tant que personnalité, lui a donné le sentiment de rupture avec soi. Il se voit non seulement démuni de ses rêves et de ses hauts désirs, 
mais il regrette également tout ce qu'il a sacrifié :

[...] je me reprochai non seulement d'avoir quitté mon pays, détruit mes papiers, perdu mon ami, mais de ne plus supporter personne; alors que mon but restait de trouver ma place dans la société européenne, je refusais celle qu'on m'offrait, je préférais m'enliser, dévisser... Prochaine étape, la folie sans doute ?» (Schmitt, 2008, p. 95).

L'erreur stratégique de Saad ainsi que de la plupart des immigrants c'est qu'ils pensent qu'en changeant leur espace, ils peuvent résoudre leurs problèmes. Saad reproche à son père son choix; le choix de l'Irak comme son pays :

Tes gendres et tes petits-enfants sont morts [...], tes filles ont été couvertes des voiles du deuil avant l'âge, [...], à cause de l'endroit où tu as bâti ta toile. Moi, je ne veux pas offrir le chaos à mes enfants. »(Schmitt, 2008, p. 81).

Même Michel Foucault dans «Des espaces autres » affirme que l'époque actuelle est davantage déterminée par l'espace que par le temps. Les idéalisations et les préjugés avaient éloigné Saad de la réalité : «Mon désir de partir me donnait une énergie inépuisable, une force constante, renouvelée, plus grande que moi, capable d'outrepasser toute limite, y compris celle du bon sens. » (Schmitt, 2008, p. 71).

De l'autre côté, Schmitt en donnant la parole à cet immigré clandestin le rend doublement responsable de son destin. En effet, bien que tout au long du roman, Schmitt défende toujours indirectement les droits des immigrés, en fin de compte, le responsable premier de tous ces problèmes est Saad lui-même. Saad avait en Egypte aussi bien qu'en Italie des occasions pour être heureux et avoir une vie tranquille : " Pourquoi ai-je quitté l'Egypte plutôt que de $\mathrm{m}^{\prime} \mathrm{y}$ installer ? [...] si j'avais abandonné au Nil mes envies d'Occident, j'aurais pu me construire une situation solide en m'épargnant des années de souffrance et d'humiliation. » (Schmitt, 2008, p. 71).

Il a beau fuir son pays, ce qu'il a trouvé au bout du chemin est tout sauf le rêve qu'il souhaitait : «De temps en temps [...], je m'étonnais des caprices du destin ; durant quelques secondes, je réalisais que j'avais quitté l'Irak et ses injustices pour me retrouver à Naples exploité par la mafia. » (Schmitt, 2008, p. 106)

Saad qui a voulu quitter son pays n'importe comment et à n'importe quel prix en acceptant tous les obstacles, est maintenant arrivé au bout d'espoir, il est pris d'un sentiment de remords. La situation embarrassante dans laquelle il se trouve a transformé ses rêves en cauchemars, il est devant des illusions jamais réalisées.

Cela montre l'apogée du pouvoir de l'art de Schmitt qui réussit à établir l'équilibre et à mettre en évidence différents aspects de la migration. En même temps qu'il invite ses lecteurs à un comportement digne de l'humanité à l'égard des immigrés, avec la déclaration de ces sentiments de regret qu'éprouve Saad, il incite les candidats au voyage clandestin à s'éloigner des illusions et à décider avec le bon sens, non avec les rêve ou les idéaux en tête.

\section{CONCLUSION}

Face à un monde dominé par les guerres, le terrorisme et les violences, où le mal semble l'emporter sur le bien, le rôle de l'écrivain devient de plus en plus important. Sa plume peut devenir une arme pour lutter contre les tares de la société. A travers son écriture, il peut inciter ses lecteurs à une prise de conscience des problématiques du monde, et les encourager à s'engager dans la lutte contre la violence et l'injustice sociale.

Dans cette étude, les différentes étapes de notre analyse ont montré comment l'écriture de Schmitt se révèle une prise de position engagée dans la mise au jour des troubles qui ont secoué l'Irak, des troubles que l'on peut voir encore dans d'autres pays, par exemple en Syrie. C'est d'une manière oblique qu'il a tenu un discours d'actualité, et qu'il a invité ses lecteurs à en mesurer la gravité. En effet, c'est avec un sens d'humour qu'il a abordé la problématique de la guerre et ses effets néfastes; ainsi que l'immigration clandestine qui reste un sujet polémique de notre temps. Schmitt, en parodiant la structure mythique traditionnelle de l'épopée odysséenne, a diminué la charge pathétique de situations inquiétantes des immigrés clandestins pour nous conduire vers une 
réflexion plus profonde sur notre propre condition humaine. Et c'est à travers des discours philosophiques qu'il essaie d'arracher ses lecteurs d'une certaine indifférence: Avons-nous vraiment plus de mérite que ces clandestins?

En effet, l'idée de faire de ce jeune garçon irakien un héros courageux et intelligent qui risque sa vie pour trouver une meilleure vie, c'est la mise en question de la politique des administrations officielles qui sont pires que les pires tyrans. Tout en faisant mine de respecter les lois, on broie les droits. Notre Ulysse n'est qu'une victime. La victime de l'égoösme et de l'hypocrisie des gens qui n'ont jamais goûté la mauvaise situation des démunis comme Saad. Comme on l'a constaté, Schmitt est sans cesse en train de critiquer la situation présente, mais il ne se contente pas de critiquer seulement, il essaie aussi de proposer des solutions pour résoudre ce problème sociopolitique. Ce qui rend efficace sa critique et sa suggestion pour résoudre ce problème, c'est qu'il les exprime d'une manière indirecte et implicite. Et d'autre part, ses idées pour résoudre ce problème sont exprimées sous la forme du souhait des personnages du roman dont la plupart sont des immigrés. Ce que l'on peut déduire à travers notre étude est qu'il faut tenter avant toute chose de donner à tout homme la possibilité de vivre dans le respect de sa dignité et de sa liberté de pensée, dans son pays. Tout cela fait partie des droits dont étaient dépourvus Saad et ses compatriotes. $C^{\prime}$ était justement ce manque qui les a conduits vers la migration.

Par ailleurs, ce récit de migration essaie de répandre la pensée de fraternité. $C^{\prime}$ est exactement ce qui est exprimé par l'Assemblée générale des Nations unies : tous les êtres humains sont doués de raison et de conscience, et doivent agir les uns envers les autres dans un esprit de fraternité. Schmitt en laissant la parole aux plus démunis ; « les immigrés », prouve sa solidarité et agit ainsi dans un esprit de fraternité. Maintenant, c'est à nous de tenter, chacun à sa manière, de donner le droit à tout homme de vivre dans une société établie sur la paix et la justice. « $C^{\prime}$ est pourquoi le bout d'un texte n'est pas sa fin, mais l'attente de sa lecture, le début de son pourquoi, de son vers quoi ». (Duchet, p. 1971)

\section{REMERCIEMENTS}

Nous remercions toute personne ayant pris part à la lecture et à l'amélioration du présent article, y compris toute l'équipe de la revue FRANCISOLA qui nous a permis de nous corriger et d'adapter notre travail aux critères recherchés.

\section{RÉFÉRENCES}

Albert, C. (2005). L'immigration dans le roman francophone contemporain. Paris: Karthala.

Manço, A. (2006). Processus identitaires et intégration. Paris: L'Harmattan.

Dapsens, S. (2005). Les causes de migratio: pistes d'analyse et d'action. Centre Avec.

Duchet, C. (1971). Pour une sociocritique, ou variations sur un incipité: Revue Littérature, $1: 5-14$

Gauthier, M. (1997). Pourquoi partir? La migration des jeunes d'hier et d'aujourd'hui, Sainte-Foy. Presses de l'Université Laval-IQRC.

Michel, F. (2009). Le corps utopique; suivi de Les hétérotopies. Paris: Éditions Lignes.

Sapiro, G. (2007). Pour une approche sociologique des relations entre littérature et idéologie. Repéré à http://journals.openedition.org/cont extes/165 ; DOI

10.4000/contextes.165

Sartre, J-P. (1948). Situations. Tome II. Paris: Gallimard.

Sayad, A. (1984). Tendances et Courants des Publications en Sciences Sociales sur l'Immigration en France Depuis 1960. Revue Current Sociology, 32: 219-304.

Sayad, A. (1999). La double absence. Des illusions de l'émigré aux souffrances de l'immigré. Paris : Seuil.

Schmitt, E-E. (2008). Ulysse from Bagdad. Paris: Éditions Albin Michel.

Schneider, A. (2013), La Littérature de jeunesse migrante. Récits d'immigration de l'Algérie à la France, Paris, L'Harmattan, coll. «Espaces littéraires». 